\title{
The Nature of the Increased Peripheral Resistance in Hypertension*
}

\author{
James Conway, M.D., PH.D. \\ Ann Arbor, Michigan
}

\begin{abstract}
A Lthough it has long been known that the elevation of blood pressure in hypertension is the result of increased peripheral resistance, the means by which this abnormality occurs has not been established. It has generally been assumed that it is due to an increase in muscle tone but the absence of a method for measuring the degree of vasomotor tone has made it impossible to verify this.
\end{abstract}

\section{VASOMOTOR TONE}

In any vascular bed there are two variables governing the relation between pressure and flow. The first is the basic structure of the vas-

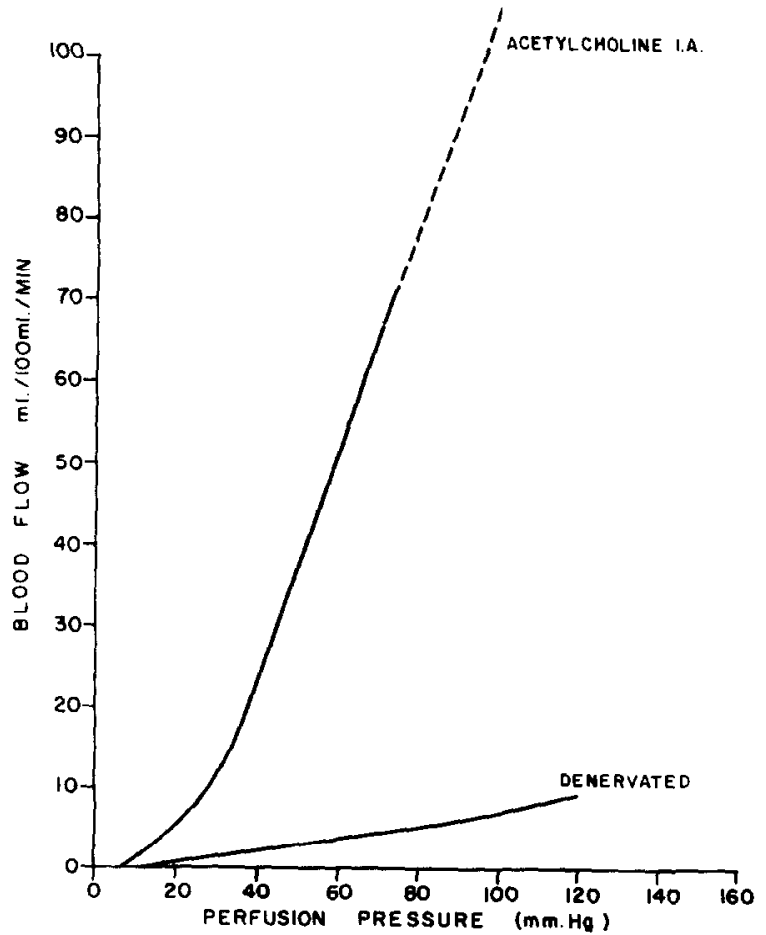

cular tree supplying the area, that is, the number of blood vessels and their cross-sectional area. The second is the degree to which the smooth muscle in these vessels is actively contracting to produce what is known as tone. That the blood flow depends on these two variables is demonstrated in Figure 1, which shows the pressure-flow curves obtained from the hind limbs of two dogs. The two preparations in the denervated state had approximately the same rate of blood flow but in one of them (left) the removal of vascular smooth muscle tone by an intra-arterial infusion of supramaximal doses of acetylcholine led to a twentyfold increase in

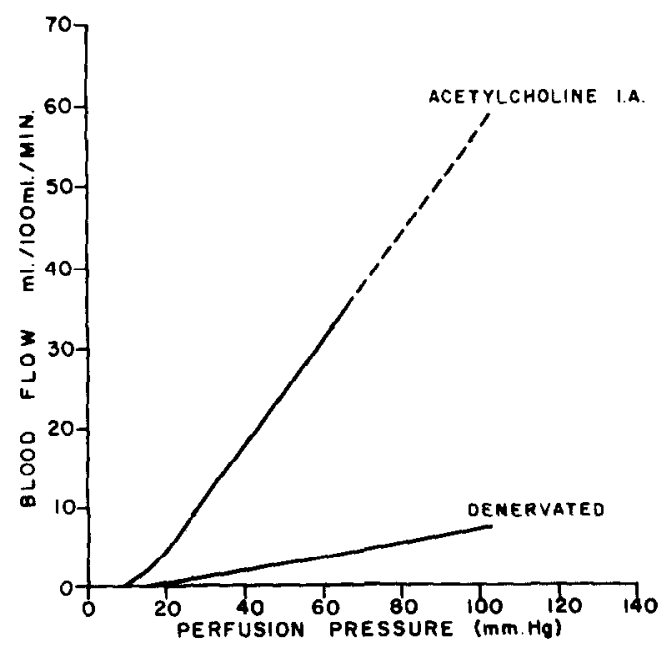

Fig. 1. Pressure-flow curves obtained from the denervated hind limb of two dogs with similar flow rates. Note the difference in flow after maximal dilation of the bed by acetylcholine. See text.

* From the Department of Medicine, The University of Michigan, Ann Arbor, Michigan. 


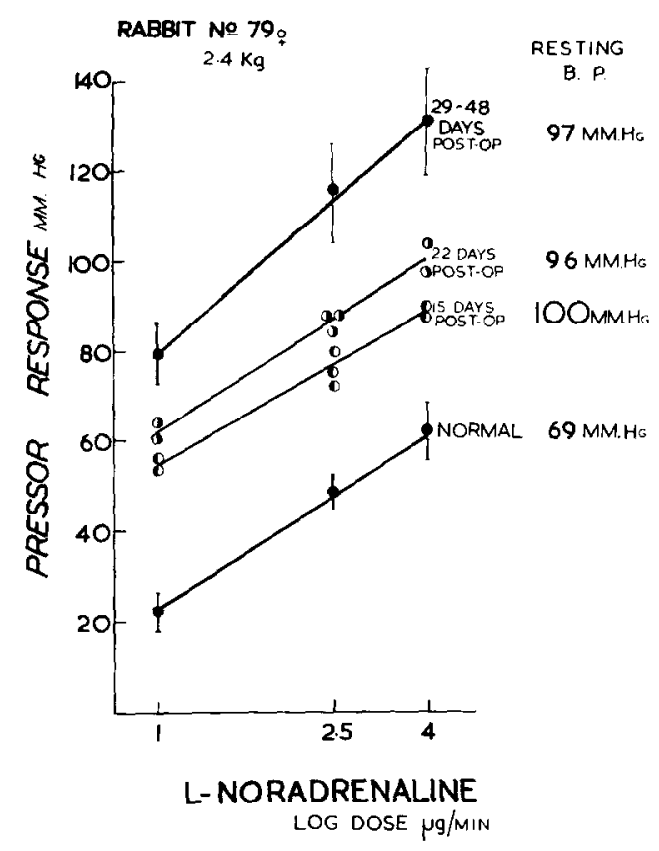

FIc. 2, Repeated dose response curves to norepinephrine in a rabbit in the normal state and again after the development of hypertension. Each study was performed after the animal had been given a large dose of hexamethonium.

flow. In the animal shown on the right, release of vasomotor tone led to a much smaller increase. In the second preparation, therefore, there was a large element of residual or structural resistance, whereas vascular smooth muscle played a smaller part in the regulation of flow. The separation of these two variables in hypertension has never been satisfactorily achieved.

\section{Structural Factors in Hypertension}

The possibility that structural factors might be involved in the changes in resistance in the state of hypertension was suggested by a study of the increased reactivity to administration of catecholamines in animals with hypertension. ${ }^{1}$ The response of the blood pressure in a rabbit to administration of norepinephrine in the normal state and again after the development of hypertension is shown in Figure 2. The increase in pressor effect occurs gradually over the course of a month after the development of hypertension, during which time the elevated level of the blood pressure has not changed. The increased reactivity is therefore not a passive reflection of the hypertensive state but develops independently. Further investigation of this phenomenon revealed that it was not confined to norepinephrine but was a non-specific response to many

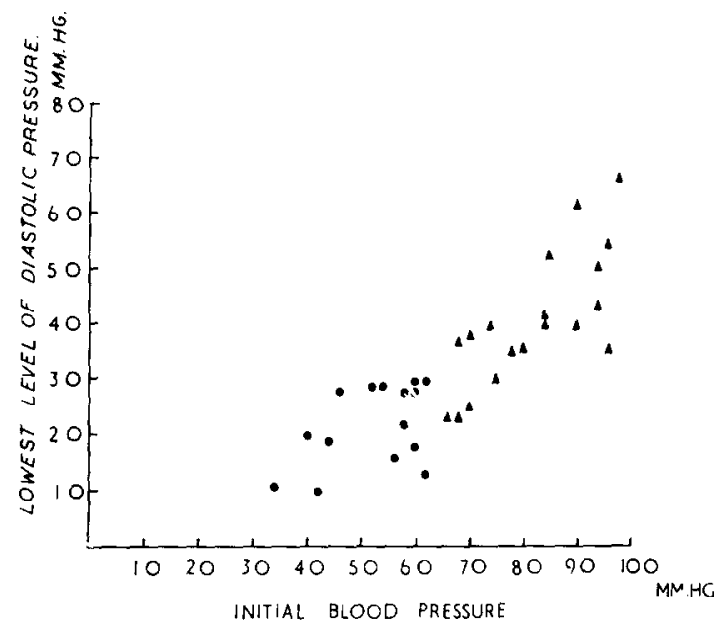

FIG. 3. Structural resistance and hypertension. The lowest level of bloud pressure produced by supramaximal intravenous doses of nitroglycerin in eight rabbits before and after the development of renal hypertension. normal. $\Delta=$ after operation for the production of hypertension.

pressor agents and also to depressor drugs. ${ }^{2,3}$ It was also found that the duration of the response to administration of norepinephrine or nitroglycerin was not prolonged in the hypertensive state as might be expected if there were a true increase in sensitivity of vascular muscle to these drugs. Injection of nitroglycerin provided further interesting and unexpected results in animals with hypertension. However large the dose of nitroglycerin, the blood pressure could never be reduced to the same level in hypertension as it could in the normal state (Fig. 3).

These results suggested the possibility that increased reactivity in hypertension could he explained on a mechanical basis if there were an increased thickness of the internal layers of the arteriolar wall. This would give increased vascular responses to drugs, yet it would be a non-specific effect and the duration of the action of the drugs would be unchanged.

Plethysmographic Studies: At this time it was demonstrated by Folkow ${ }^{5,6}$ that in hypertension in human beings the blood vessels of the forearm could not be dilated to the same extent in patients with hypertension as in normotensive subjects. Since the concept of an increased structural resistance would have an important bearing on our understanding of the nature of hypertension, further investigation of this possibility has been undertaken in eleven normal subjects and twenty-seven patients with hyperten- 


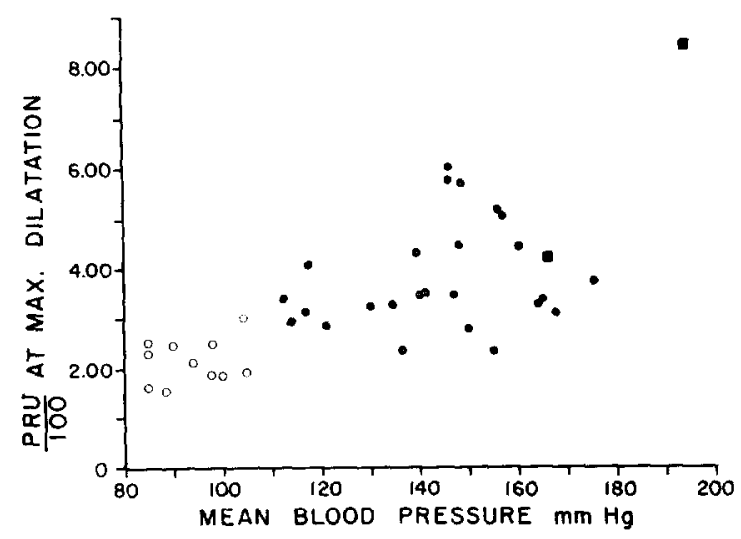

Fig. 4. Structural resistance and hypertension. The relationship between blood pressure and minimal structural resistance in the forearm vessels of normal subjects and patients with hypertension. Structural resistance, measured after maximal dilation, has been produced in the forearm vessels by exercise and ischemia of eight minutes' duration. $O=$ normal subjects. $O=$ essential hypertension, $\boldsymbol{\square}=$ renal hypertension.

sion. Blood flow in the forearm was measured by a Whitney strain gage plethysmograph ${ }^{7}$ after maximal dilation was produced by exercise and ischemia of eight minutes' duration. The greatest blood flow recorded after ischemia, taken with the level of blood pressure, gave the structural resistance. The mean resistance in the normal subjects was 2.20 peripheral resistance units per $100 \mathrm{cc}$. of tissue, whereas only 2 patients with hypertension had this level. The relation between the structural resistance and the severity of hypertension is shown in Figure 4. Thus it can be seen that the structural resistance rises roughly in proportion to the level of blood pressure.

It is possible that this increased structural resistance is the consequence of hypertension and not associated with its initiation. If this were so the structural changes demonstrated here would he an indication of the accelerated arteriosclero- sis associated with hypertension. Preliminary studies, however, show that the development of structural changes in hypertension is not a continuing process, since there is no demonstrable association between the level of structural resistance and the duration of hypcrtension.

\section{Summary}

Studies on hypertension in both human beings and animals have shown an increased response to vasopressor and vasodepressor agents. This increased reactivity, as well as the incompleteness of vasodilatation by large doses of vasodepressor agents, is believed to be due to structural changes in the vascular tree. Plethysmographic studies have demonstrated an increased structural resistance in blood vessels of the forearm in patients with hypertension as compared with normal subjects.

\section{REFERENGES}

1. Conway, J. The behavior of the blood pressure in normal and hypertensive rabbits in response to l-noradrenaline and to ganglion block by hexamethonium or pentamethonium. J. Physiol., 127: 69, 1955.

2. Conway, J. The direct and reflex effects of injections of nitroglycerine and l-noradrenaline in normal and hypertensive rabbits. Clin. Sc., 14:625, 1955.

3. Brown, G. M. and Maegraith, B. G. Characteristics of the circulation of hypertensive rabbits. J. Physiol., 99: 304, 1941.

4. Cionway, J. In: Symposium on Hypotensive Drugs, p. 193. Edited by Harington, M. London, 1956. Pergamon Press.

5. Folkow, B. In: Symposium on Hypotensive Drugs, p. 163. Edited by Harington, M. London, 1956. Pergamon Press.

6. Folkow, B., Grimby, G. and Thulseius, O. Adaptive structural changes of the vascular wall in hypertension and their relation to the control of the peripheral resistance. Acta. physiol. scandinat., $44: 225,1959$.

7. Whitney, R. J. The measurement of volume changes in human limbs. J. Physiol., 121:1, 1953. 